\title{
QUALITATIVE SIMULATION OF CONSTRUCTION PERFORMANCE USING FUZZY COGNITIVE MAPS
}

\author{
Manjula Dissanayake \\ Simaan M. AbouRizk \\ Hole School of Construction Engineering \\ Department of Civil and Environmental Engineering \\ University of Alberta \\ Edmonton, AB, T6G 2G7, CANADA.
}

\begin{abstract}
The construction process is subject to an array of influences, both from internal and external environments, which makes the process performance uncertain and difficult to predict. To deal with uncertainty, simulation is widely applied in construction process modeling. Most of the applications, however, are restricted to quantitative models due to limited qualitative simulation capabilities.

In this paper, Fuzzy Cognitive Maps (FCM) are proposed as a means for modeling and controlling complex construction performance-related problems qualitatively. They provide an effective tool for answering what-if questions during the construction planning phase.
\end{abstract}

\section{INTRODUCTION}

Construction performance-related problems are generally complex, ill-defined, and dynamic in nature. Most of the related data are available in a qualitative form rather than quantitative, mainly in the form of cause/effect-type relationships that are expressed in liguistic terms. Hence, in most cases, developing precise models for reasoning construction performance are impractical. The aforementioned characteristics of the problem bring up the need for approximate reasoning methods that will handle imperfect information.

The Fuzzy set theory (Zadeh 1965) based decision support systems are commonly used in approximate reasoning. However, decision support systems, including those incorporating fuzzy rules and inferencing, suffer from the difficulty of eliciting if-then rules and maintaining the resulting rule-bases. This is mainly because not allexpert knowledge can be captured in the form of fuzzy production rules.

Fuzzy Cognitive Maps (FCMs) can avoid many of the knowledge-extraction problems, which are usually posed by rule-based systems and allows modeling feedback (cycles) in complex domains (Pearl 2000). FCMs are fuzzy-graph structures that provide an expressive and flexible method of capturing and representing complex relationships in an intuitive manner. They have been developed in the world of soft computing, combining the robust properties of fuzzy logic and neural networks. FCMs have been sucessfully used to model complex systems in a variety of scientific areas (Aguilar 2005).

Once constructed, FCM models make the cause/effect relationships apparent; allowing for the performing of a qualitative simulation of the system and experiment with the model in order to carry out a what-if analysis during the construction planning phase.

This paper proposes a qualitative simulation framework using FCMs that is able to perform a qualitative representation of construction performance factors and to conduct qualitative simulation in order to execute the whatif analysis to facilitate construction project planning and control. This paper is organized as follows. Section 2 presents a brief overview of the theoretical background of FCM models, and their inference mechanism. Section 3 discusses the FCM model development process, and the issues to be considered during FCM construction. In Section 4, a case study is presented along with a description of a prototype system developed to design and implement qualiative simulation using FCMs. Section 5 concludes the paper by making suggestions for future research.

\section{FUZZY COGNITIVE MAPS: BASICS}

The structure and behavior of FCMs are briefly described in this section.

\subsection{Theoretical Background}

A cognitive map (CM) is a representation of an individual's (or group of individual's) knowledge of their spatial environment. Axelrod (1976) described CM in a logical manner, primarily focusing on the analysis of the effect of policy alternatives upon valued goals. Kosko 


\section{Dissanayake and AbouRizk}

(1986) developed the fuzzy extension of the CM, namely FCMs, to represent causal reasoning.

FCMs are fuzzy-signed diagraphs with feedback that describes the causal links between concepts (Kosko 1986). An FCM consists of nodes $(\mathrm{Ci})$ and edges $\left(e_{\mathrm{ij}}\right)$, representing concepts (variables) and the causal relation between nodes, respectively. The nodes $\left(C_{i}\right)$ are fuzzy sets (Kosko 1986) and, hence, take the values in $[0,1]$. $e_{\mathrm{ij}}$ can accept the values $[-1,1]$. The positive edge $\left(e_{\mathrm{ij}}>0\right)$ represents the exhibitory causal relations between nodes, and the negative edge $\left(e_{\mathrm{ij}}<0\right)$ represents the inhibitory causal relations between nodes. For example, as shown in Figure $1, C_{2}$ has an exhibitory causal relationship with $C_{1}$, i.e., increase in construction delays leads to the increase in cost overrun. Conversely, $C_{4}$ has an inhibitory causal relationship with $C_{3}$, i.e., labour wage skilled labour shortages. $e_{\mathrm{ij}}=0$ indicates no relationship between concepts $\mathrm{i}$ and $\mathrm{j}$.

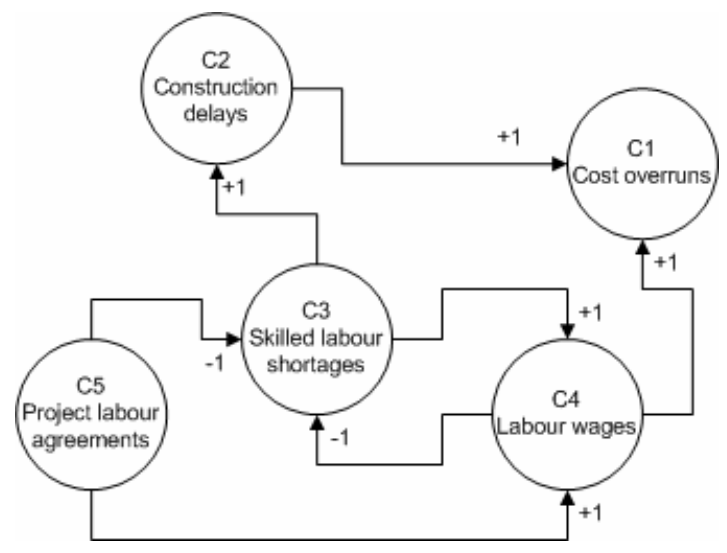

Figure 1: A simple FCM concerning cost overruns of a construction project with 5 concept nodes. Edges show the directed causal flow between nodes.

An FCM with $n$ nodes has $n^{2}$ edges. An adjacency matrix E can represent the connectivity of the FCM (Dikerson and Kosko 1997):

$$
\mathrm{E}=\left[\begin{array}{ccc}
\ldots & \ldots & \ldots \\
\ldots & \mathrm{e}_{\mathrm{ij}} & \ldots \\
\ldots & \ldots & \ldots
\end{array}\right]
$$

where $e_{\mathrm{ij}}$ is the value of the edge from node $\mathrm{i}$ to node $\mathrm{j}$. As suggested in (Dikerson and Kosko 1997) the system nonlinearly transforms the weighted input to each node $C_{\mathrm{i}}$ :

$$
C_{i}\left(t_{n+1}\right)=S\left[\sum e_{k i}\left(t_{n}\right) C_{k}\left(t_{n}\right)\right]
$$

Here $S(x)$ is a bounded signal function.

For bivalent nodes:

$$
\begin{aligned}
& \mathrm{S}_{\mathrm{i}}\left(\mathrm{y}_{\mathrm{i}}\right)=1, \mathrm{x}_{\mathrm{i}}>0 \\
& \mathrm{~S}_{\mathrm{i}}\left(\mathrm{y}_{\mathrm{i}}\right)=0, \mathrm{x}_{\mathrm{i}} \leq 0
\end{aligned}
$$

The sigmoid function

$$
\mathrm{S}(\mathrm{y})=\frac{1}{1+\mathrm{e}^{-\mathrm{c}(\mathrm{y}-\mathrm{T})}}
$$

with large $\mathrm{C}>0$ approximates a binary threshold function.

The state of the FCM at time $t$ can then be represented by the vector function $\mathbf{C}_{i}(\mathrm{t})=\left[\mathrm{C}_{1}(\mathrm{t}), \ldots, \mathrm{C}_{\mathrm{n}}(\mathrm{t})\right]$ - indexed family of fuzzy sets- and thus a point in the fuzzy hypercube $I^{\mathrm{n}}=[0,1]^{\mathrm{n}}$.

\subsection{Causal Knowledge-based Inference}

Since FCMs are dynamic systems, their equilibrium states are limit cycles. The limit cycle or hidden pattern is the FCM inference (Kosko 1986). The output equilibrium is the answer to the causal what-if questions.

\section{DEVELOPMENT OF FCMS}

FCMs can be used to exploit the causal knowledge and experience, which has been accumulated over a certain period on a complex operation. FCMs store domain knowledge in nodes and edges of the network. The most crucial step of the network design process is the knowledge representation Schneider et al. (1998), during which the expert's opinion on the problem scenario is exploited. The typical steps followed to develop FCMs are:

(1). Identification of concepts $\left(C_{\mathrm{i}}\right)$-i.e., issues, factors, variables, and goals that influence the problem.

(2). Identification of causal relationships among these concepts.

As identified in Schneider et al. (1998), the FCM literature is mainly focused on using FCMs rather than their construction. This section gives a description of the FCM model development process.

\subsection{Concept Identification}

The concepts best describe the scenario; the direction and the grade of causality between the concepts should be determined at this stage.

Different methods of deriving cognitive maps from interviews/questionnaires and textual descriptions given by experts', are described by Axelrod (1976) . It is obvious that the map represents a subjective opinion on the given problem scenario.

In the case where multiple FCMs are availablerepresenting different expert's opinions-the FCMs can be combined to form a new augmented FCM (AFCM). As given in Dikerson and Kosko (1997), the AFCM includes the union of the causal concepts of all FCMs. The combined edge matrix is given by:

$$
F=\sum_{I}^{N} w_{i} E_{i}
$$


where $F$ is the sum of edge matrices, $w_{i}$ are positive weights for the $i^{\text {th }}$ expert, $E_{i}$ is the $i^{\text {th }}$ expert's cognitive map in matrix form, and $N$ is the number of contributing experts.

As described before, the nodes $\left(C_{i}\right)$ are fuzzy sets, hence, the concept values that are numerical measurements need to be converted into fuzzy sets. Membership degrees of each causal concept can be derived by identifying the maximum $(\mu=1)$ and minimum $(\mu=0)$ measurements and applying to the equation:

$$
\mu\left(\mathrm{c}_{\mathrm{i}}\right)=\frac{\mathrm{c}_{\mathrm{i}}-\min (\mathrm{C})}{\max (\mathrm{C})-\min (\mathrm{C})}
$$

Where applicable, to facilitate a more reasonable representation of the state of the concept, a threshold (T) can be applied so that the concepts that possess values $\mathrm{c}_{i}>\mathrm{T}$ can assign a value $\mu=1$ and conversely, where $\mathrm{c}_{i}<\mathrm{T}$ can assign the value $\mu=0$.

\subsubsection{Determining the Direction of Causality}

Identifying causality is critical to predicting future events with FCMs. As identified by Pearl (2000), most theories of causation invoke an explicit requirement that a cause precedes its effect in time (i.e., temporal precedence). For example, as shown in Figure 1, $\mathrm{C}_{3} \rightarrow \mathrm{C}_{2}$ implies skill labour shortages precede construction delays. Note that unlike correlation, the causal relationship is unsymmetrical.

In FCMs, the causality is determined by the opinion of the experts involved in the construction of the FCM.

\subsubsection{Determining Direct vs. Inverse Relations}

In general, edges in a FCM have values in the interval [1,1]. High (absolute) values of causality signify strong cause-effect relationships between the concepts. The relationship $c_{i}(+1) \rightarrow c_{j}$ indicates that:

If $\mathrm{c}_{\mathrm{i}}$ changes very positive/negatively, then the impact on $\mathrm{c}_{\mathrm{j}}$ is very positively/ negatively.

If $c_{i}$ changes a little positively/negatively, then the impact on $c_{j}$ is a little positively/negatively.

Thus, FCM is able to, process all these rules in parallel and represent as a list of rules.

The causal relationships can take various functional forms (Koulouriotis et al. 2003) such as the linear, the stepwise, the sigmoid, the Gaussian and even the exponential family of curves. Assigning a crisp value for causality (in conventional FCMs) implies a linear causal relationship between concepts. The selection of the functional form of the causality depends on the expert's opinion on the causality of the concepts in query.

\section{CONSTRUCTION PERFORMANCE SIMULATION}

Construction management involves scheduling and coordination of construction activities, evaluating time and cost consequences of construction decisions, contract negotiations, and so on. The construction managers' goals are to control projects, be sure they come in on budget, avoid any cost overruns, and ensure that the various stages of the construction process are completed in a timely and seamless fashion. To achieve the above goals, at the planning stage of construction projects, construction mangers analyze different what-if scenarios to identify the impact on project performance goals, before making any decisions.

Simulation is a technique for performing these "whatif" analyses. In construction planning, simulation is commonly used for analyzing risk events associated with the planning of construction projects (Hajjar and AbouRizk 2002). Earth moving (e.g., Smith et al. 1995), aggregate production (e.g., Hajjar and AbouRizk 2002), tunnel construction (e.g., Ruwanpura et al. 2001), are few applications of risk analysis of construction operation using discrete event simulation. One commonality in these simulation applications is the use of quantitativeprobabilistic approach to model construction performance at the activity level. The focus of this paper is rather qualitative, at a different reasoning level, i.e., at a strategic level where construction managers are given the opportunity to reason with the causal knowledge of key performance factors and indicators.

\subsection{User Mental Models: Knowledge Representation}

As explained in Section 2, in FCMs, the user represents his or her knowledge about a particular scenario using concepts and edges. When construction-related problem scenarios are analyzed, concepts can be categorized into three (1) external, (2) internal, and (3) goal concepts, as shown in Table 1.

The external concepts represent the factors that are beyond the control of the user (e.g., construction manager), whereas the internal concepts represent the factors that can be controlled by the user.

In the construction domain, concept identification can be carried out by the construction project management team, using one of the group consensus approaches (e.g., facilitated brainstorming, nominal group technique, or Delphi technique), preferably on a project by project basis. 


\section{Dissanayake and AbouRizk}

Table 1: Concept categories and examples of construction performance concepts

\begin{tabular}{lll}
\hline External Concepts (Causes) & Internal Concepts (Causes/effects) & Goal Concepts (Effects/Causes) \\
& & \\
\hline $\begin{array}{l}\text { Scope changes by client } \\
\text { Design changes }\end{array}$ & Lack of workface planning & Project cost (e.g., Cost Performance Index) \\
$\begin{array}{l}\text { Regulatory changes } \\
\text { Lack of design coordination } \\
\text { Bad weather }\end{array}$ & Lack of work experience and skills & Schedule (e.g., Schedule variance) \\
& Lack of worker motivation (e.g., Cost of rework \%) \\
& Congested work areas & Labour productivity \\
Unforeseen site conditions & Worker safety (e.g., Lost Time Incidents- \\
& $\begin{array}{l}\text { Non availability of } \\
\text { equipments/tools }\end{array}$ & \\
\hline
\end{tabular}

\subsection{System Prototype-FuzCog}

To facilitate the development of FCMs, a prototype system (FuzCog) is developed in an existing simulation platform named Simphony (Hajjar and AbouRizk 1999). FuzCog is developed as a template in Simphony so that user can create any number of FCMs at different reasoning levels. As shown in Figure 2, FuzCog consists of three main areas, (1) FCM modeling elements: concepts, causal links, and membership functions, (2) FCM Model: created by the user using modeling elements, and (3) Simulation results.

The modeling elements allow the user to define concepts, their initial state (value), and relationship with other concepts. The concept states after each simulation runs are shown in the results window.

\subsection{An Example: Reasoning Schedule Performance}

An example problem scenario is given below to explain the concepts discussed above. The schedule performance (duration) is selected as the goal concept. Table 2 shows a list of concepts/variables that affect the construction project duration (goal concept). The causal model based on those selected variables is graphically shown in Figure 3. The interrelationships between the concepts are shown in Figure 4.

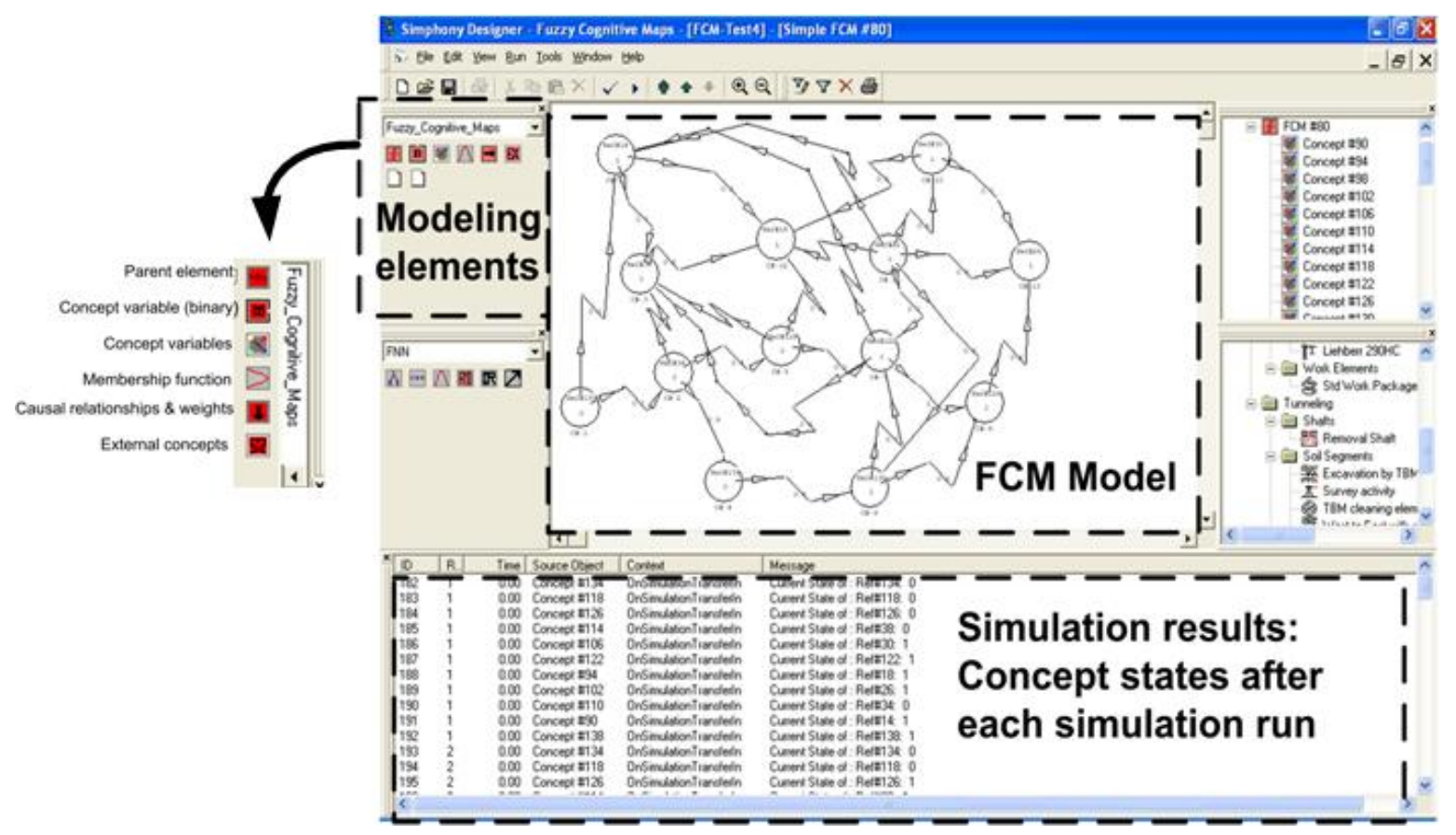

Figure 2: FuzCog User Interface 


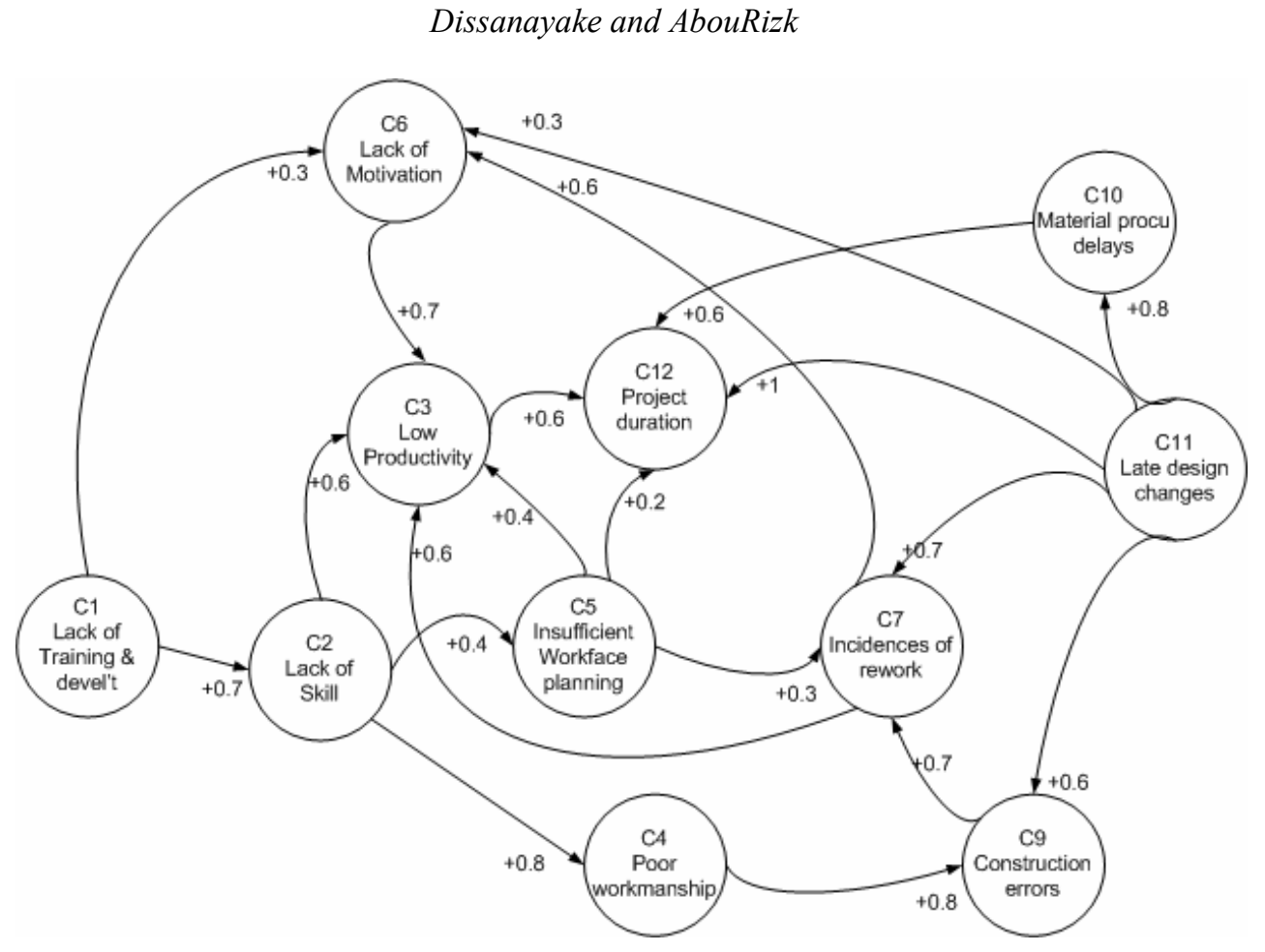

Figure 3: Causal Model

Table 2: Concept categories and examples of construction performance concepts

\begin{tabular}{llll}
\hline \multicolumn{3}{l}{ Concept } \\
\hline C1 & Lack of training and skill development \\
& opportunities & & \\
C2 & Lack of skill & \\
C3 & Low labour productivity & \\
C4 & Poor workmanship & \\
C5 & Insufficient workface planning & \\
C6 & Lack of motivation & \\
C7 & Incidents of rework \\
C9 & Construction errors \\
C10 & Material procurement delays & \\
C11 & Late design changes \\
C12 & Project duration (goal concept) & \\
\hline
\end{tabular}

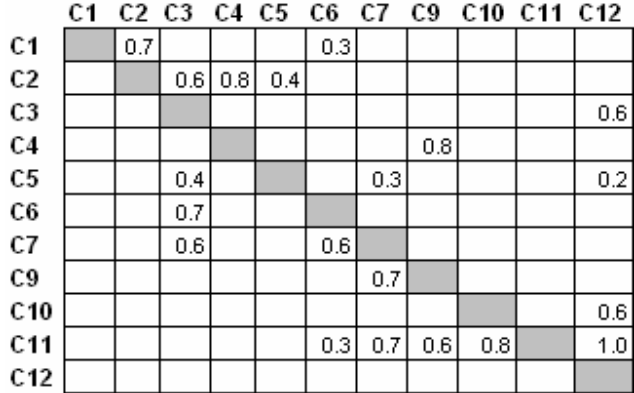

Figure 4: The Edge Matrix
Assuming the linear relationships between concepts and the binary threshold functions, the following three scenarios were derived to explain the concepts further. Summary results are given in Figure 5.

\subsubsection{Case 1: Effect of Late Design Changes}

Using equations (2) and (3), inferences have made to identify the effect of late design change on the identified problem scenario. As shown in Figure 5.a, the FCM has come to an equilibrium state in its second simulation run. FCM stables in a limit cycle of $\mathrm{C} 3 \rightarrow \mathrm{C} 6 \rightarrow \mathrm{C} 7 \rightarrow \mathrm{C} 9 \rightarrow \mathrm{C} 10 \rightarrow \mathrm{C} 12$.

It basically gives the idea that when a contractor receives a late design change, it leads to a rework incidence and or lack of motivation of workers that leads to low productivity leading to the extension of the project duration.

\subsubsection{Case 2: Effect of Lack of Skill level of Labour}

This case shows the impact of low skilled workforce on the overall project performance. As shown on Figure 5.b, after 6 simulation runs, the FCM stabilizes to a fixed point.

Based on the FCM inference it can be concluded that the lack of a skilled workforce leads to poor workmanship, incidence of rework, low productivity, and that ultimately leads to the extension of project duration. 
a. Effect of Late design Changes

\begin{tabular}{|c|c|c|c|c|c|c|c|c|c|c|c|}
\hline Run & C1 & $\mathrm{C} 2$ & C3 & $\mathrm{C4}$ & C5 & C6 & C7 & C9 & C10 & C11 & $\mathrm{C}_{12}$ \\
\hline 0 & 0 & 0 & 0 & 0 & 0 & 0 & 0 & 0 & 0 & 1 & 0 \\
\hline 1 & 0 & 0 & 0 & 0 & 0 & 1 & 1 & 1 & 1 & 0 & 1 \\
\hline 2 & 0 & 0 & 1 & 0 & 0 & 1 & 1 & 1 & 1 & 0 & 1 \\
\hline 3 & 0 & 0 & 1 & 0 & 0 & 1 & 1 & 1 & 1 & 0 & 1 \\
\hline
\end{tabular}

b. Effect of Lack of Skill level

\begin{tabular}{c|ccccccccccccc} 
Run & C1 & C2 & C3 & C4 & C5 & C6 & C7 & C9 & C10 & C11 & C12 \\
\hline 0 & {[} & 0 & 1 & 0 & 0 & 0 & 0 & 0 & 0 & 0 & 0 & 0 & ] \\
1 & {[} & 0 & 0 & 1 & 1 & 1 & 0 & 0 & 0 & 0 & 0 & 0 & ] \\
2 & {[} & 0 & 0 & 1 & 0 & 0 & 0 & 1 & 1 & 0 & 0 & 1 & ] \\
3 & {[} & 0 & 0 & 1 & 0 & 0 & 1 & 1 & 0 & 0 & 0 & 1 & ] \\
4 & {[} & 0 & 0 & 1 & 0 & 0 & 1 & 0 & 0 & 0 & 0 & 1 & ] \\
5 & {[} & 0 & 0 & 1 & 0 & 0 & 0 & 0 & 0 & 0 & 0 & 1 & ]
\end{tabular}

c. Effect of Rework incidence

\begin{tabular}{|c|c|c|c|c|c|c|c|c|c|c|c|}
\hline Run & C1 & $\mathrm{C} 2$ & C3 & $\mathrm{C} 4$ & C5 & C6 & C7 & C9 & C10 & C11 & $\mathrm{C}_{12}$ \\
\hline 0 & {$[0$} & 0 & 0 & 0 & 0 & 0 & 1 & 0 & 0 & 0 & 0 \\
\hline 1 & 0 & 0 & 1 & 0 & 0 & 1 & 0 & 0 & 0 & 0 & 0 \\
\hline 2 & 0 & 0 & 3 & 0 & 0 & 0 & 0 & 0 & 0 & 0 & 1 \\
\hline 3 & 0 & 0 & 0 & 0 & 0 & 0 & 0 & 0 & 0 & 0 & 1 \\
\hline 4 & 0 & 0 & 0 & 0 & 0 & 0 & 0 & 0 & 0 & 0 & 0 \\
\hline
\end{tabular}

Fig.5: Summary Results

\subsubsection{Case 3: Effect of incident of rework}

Based on the results shown in Figure 5.c, it can be concluded that when there is a rework incident, it impacts the labour productivity and at the same time it has a negative impact on workers motivation, which ultimately leads to an extension of project duration.

The above example scenarios illustrate the type of inference that can be made using the qualitative simulation of FCMs. In addition to the results presented above, the states of each concept after the system stables can be used as inputs for simulation model(s) that are developed at different reasoning level(s). For example, the final state of the concept "labour productivity (C3)" can be used as an input to an activity level discrete-event simulation model to calculate the duration of a certain activity.

\section{CONCLUSIONS AND FUTURE RESERACH}

\subsection{Conclusions}

This paper presented a subjective modeling methodology to model construction performance based on FCMs. A prototype system (FuzCog template in Simphony) was developed in order to facilitate the process and design details have been described.
The proposed methodology helps construction managers represent their causal knowledge (world view) on problem scenarios in a systematic way; and quantifies the impact of management decisions and external factors on project performance. The proposed system supplements the current construction planning by conducting what-if simulations to identify the impact of different scenarios, qualitatively. Moreover, the proposed mechanism aims at simulating performance at an abstract level with imprecise concepts and their relationships.

\subsection{Future Research}

The main challenge faced while conducting the experiments described in this paper is that of building an appropriate causal model. Expert knowledge plays a key role not only in identifying the concepts and their relationships, but also in revising and fine-tuning the model. Hence, the proposed methodology can benefit by exploring efficient strategies through which to elicit expert knowledge.

Also, in addition to using FCMs as standalone qualitative simulation models, they can be combined with current construction simulation models in order to enhance the existing modeling capability with FCMs subjective modeling capabilities. Currently, work is in progress towards creating an integrated framework that facilitates the qualitative simulation with quantitative discrete-event simulation.

\section{ACKNOWLEDGEMENTS}

The authors wish to acknowledge the comments and useful suggestions offered by Drs. Witold Pedrycz, Aminah Robinson Fayek, Yasser Mohamed, and Lingguang Song.

\section{REFERENCES}

Aguilar, J. (2005). A survey about fuzzy cognitive maps papers (Invited paper). International Journal of Computational Cognition, 3(2): 27-33.

Axelrod, R. (1976). Structure of Decision: the cognitive maps of political elites. Princeton University Press, Princeton, NJ.

Dikerson, J. A., and Kosko, B. (1997). Virtual worlds in fuzzy cognitive maps. Fuzzy Engineering, B. Kosko, ed., Prentice Hall, NJ, 499-529.

Hajjar, D., and AbouRizk, S. M. (1999). "Simphony: an environment for building special purpose construction simulation tools." Proceedings of the Winter SimulationConference.

Hajjar, D., and AbouRizk, S. M. (2002). Unified Modeling Methodology for Construction Simulation. Journal of Construction Engineering and Management 128(2): 174-185.

Kosko, B. (1986). Fuzzy cognitive maps. International Journal of Man-Machine Studies, 24: 65-75. 
Koulouriotis, D. E., Diakoulakis, I. E., Emris, D. M., Antonidakins, E. N., and Kaliakatsos, I. A. (2003). Efficiently modeling and controlling complex dynamic systems using evolutionary fuzzy cognitive maps (invited paper). International Journal of Computational Cognition, 1(2): 41-65.

Pearl, J. (2000). Causality: models, reasoning, and inference. Cambridge University Press, New York, NY.

Ruwanpura, J. Y., Abourizk, S. M., Er, K. C., and Fernando, S. (2001). Special purpose simulation templates for tunnel construction operations. Canadian Journal of Civil Engineering, 28(2): 222237.

Schneider, M., Shnaider, E., Kandel, A., and Chew, G. (1998). Automatic construction of FCMs. Fuzzy Sets and Systems, 93(161), 172.

Smith, S. D., Osborne, J. R., and Forde, M. C. (1995). Analysis of earth moving systems using discrete-event simulation. Journal of Construction Engineering and Management 121(4): 388-396.

Zadeh, L. A. (1965). Fuzzy Sets. Information and Control, $8338-353$.

\section{AUTHOR BIOGRAPHIES}

MANJULA DISSANAYAKE is a research and technology transfer engineer in the Hole School of Construction Engineering, Department of Civil and Environmental Engineering at the University of Alberta. He received his Ph.D. degree in Construction Engineering and Management from University of Alberta in 2006. His research interests lay in the area of applying Computational Intelligence (CI) tools such as fuzzy logic, neural networks, and genetic algorithms, to solve real-world problems encountered in construction engineering and management. Specific areas of interests include modeling construction productivity; automated reasoning of construction performance; and modeling and simulation of construction operations. His e-mail address is

<manjulad@ualberta.ca>

SIMAAN M. ABOURIZK is a Professor in the Department of Civil and Environmental Engineering at the University of Alberta. He holds the NSERC/Alberta Construction Industry Research Chair in Construction Engineering and Management, and the Canada Research Chair in Operational Simulation. He received his BSCE and MSCE in Civil Engineering from Georgia Institute of Technology in 1984 and 1985, respectively; and his Ph.D. degree from Purdue University in 1990. His research interests focus on the application of computer methods and simulation techniques to the management of construction projects. His email address is 